\title{
Changes of $\mathrm{rScO}_{2}$ and $\mathrm{ScvO}_{2}$ in children with sepsis-related encephalopathy with different prognoses and clinical features
}

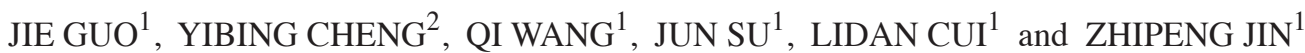 \\ Departments of ${ }^{1}$ Pediatric Intensive Care Unit (PICU) and ${ }^{2}$ Emergency, \\ Children's Hospital Affiliated to Zhengzhou University, Zhengzhou Children's Hospital, \\ Zhengzhou Children's Key Laboratory of Critical Care Medicine, Zhengzhou, Henan 450018, P.R. China
}

Received July 19, 2018; Accepted February 21, 2019

DOI: 10.3892/etm.2019.7451

\begin{abstract}
Clinical features of sepsis-associated encephalopathy in children with different prognoses were analyzed and the changes of regional cerebral oxygen saturation $\left(\mathrm{rScO}_{2}\right)$ and central venous oxygen saturation $\left(\mathrm{ScvO}_{2}\right)$ were measured. Eighty children with sepsis-related encephalopathy, admitted to the Pediatric Intensive Care Unit (PICU) of the Children's Hospital Affiliated to Zhengzhou University, were enrolled in this study and post-intensive care syndrome (PICS) scoring was performed within $24 \mathrm{~h}$ after admission. Patients were separated into groups according to the score results and treatment outcomes. Clinical features, functional tests, imaging examinations, PICS scores, and modified Glasgow Coma Scale (GCS) scores were compared among children with varying severities and prognoses. The changes of $\mathrm{rScO}_{2}$ and $\mathrm{ScvO}_{2}$ at different time-points among children with different prognoses were measured and compared. According to PICS scores, there were 8 non-critically ill children, 42 critically ill children, and 30 extremely critically ill children. Fifty-two children survived, and the survival rate was $65 \%$. Comparison of the clinical characteristics of children with different conditions showed that deep coma, generalized seizure, severe electroencephalogram (EEG) abnormalities, and the survival of children were significantly associated with the severity of disease $(\mathrm{P}<0.05)$. At the same time, compared to the survivors group, the rates of generalized seizures and severe EEG abnormalities were significantly increased in the deceased children group, while the PICS
\end{abstract}

Correspondence to: Dr Zhipeng Jin, Department of Pediatric Intensive Care Unit (PICU), Children's Hospital Affiliated to Zhengzhou University, Zhengzhou Children's Hospital, Zhengzhou Children's Key Laboratory of Critical Care Medicine, 33 Longhu Outer Ring East Road, Zhengzhou, Henan 450018, P.R. China E-mail: pd72re@163.com; jinzhipeng55@163.com

Key words: sepsis-associated encephalopathy, clinical features, $\mathrm{rScO}_{2}, \mathrm{ScvO}_{2}$, prognosis and GCS scores were significantly decreased $(\mathrm{P}<0.05)$. $\mathrm{rScO}_{2}$ values in the deceased group were lower than those in the survival group at different time-points, but the differences were not significant $(\mathrm{P}>0.05)$. On the contrary, $\mathrm{ScvO}_{2}$ values were significantly higher in the deceased group than those in the survivors group $(\mathrm{P}<0.05)$. The lower the PICS and GCS scores in children with sepsis-related encephalopathy, the more serious the condition, and the more likely to develop disturbance of consciousness, epileptic seizures, and abnormal EEG changes. Whereas, changes of $\mathrm{ScvO}_{2}$ are closely related to prognosis.

\section{Introduction}

Sepsis, a disease caused by infection in any part of the body, can eventually lead to systemic inflammatory reactions and high morbidity and mortality $(1,2)$. Sepsis-related encephalopathy refers to brain dysfunction caused by sepsis reactions. Sepsis-related encephalopathy is often reversible, and its pathogenesis remains unclear. It is generally believed that sepsis-related encephalopathy is closely related to abnormal neurotransmitters, cerebrovascular dysfunction, and inflammatory injury. In spite of the advanced anti-infective technology and the up-to-date organ support technology, the mortality rate of sepsis is still as high as $3.5 \%$, and the cost of treatment is high, which seriously affects people's life quality $(3,4)$. Therefore, the early diagnosis and treatment of sepsis is particularly important. The key to early detection is the monitoring of the disease status and some important indicators, and the key to treatment is the control of infection. Central venous oxygen saturation $\left(\mathrm{ScvO}_{2}\right)$ can be used for targeted screening of patients with early sepsis (5). In addition, a study has found that regional cerebral oxygen saturation $\left(\mathrm{rScO}_{2}\right)$ is closely related to $\mathrm{ScvO}_{2}(6)$. The present study included 80 children with sepsis-related encephalopathy who were admitted to the Pediatric Intensive Care Unit (PICU) of the Children's Hospital Affiliated to Zhengzhou University (Zhengzhou, China) from January 2015 to January 2018. Clinical data of the patients were collected and the changes of $\mathrm{ScvO}_{2}$ and $\mathrm{rScO}_{2}$ among children with different prognoses were compared. 


\section{Patients and methods}

Clinical data. Eighty children with sepsis-related encephalopathy, admitted to the PICU of the Children's Hospital Affiliated to Zhengzhou University from January 2015 to January 2018, were enrolled. Inclusion criteria: patients who met the diagnostic criteria for sepsis in children established by the International Children's Sepsis Conference (7). Patients with sepsis-associated encephalopathy were selected according to their symptoms. Exclusion criteria: patients with central nervous system infection, patients with traumatic brain injury, and patients with uremia encephalopathy and hepatic encephalopathy. The infection sources were located in different parts; there were 28 cases of lung infection, 37 cases of sepsis, 12 cases of intestinal infection, and 3 cases of skin and soft tissue infection. This study was approved by the Ethics Committee of the Children's Hospital Affiliated to Zhengzhou University. Patients who participated in this research had complete clinical data and signed informed consents were obtained from their parents.

Observation indicators. Post-intensive care syndrome (PICS) scoring and Glasgow Coma Scale (GCS) scoring were performed on children with sepsis-related encephalopathy within $24 \mathrm{~h}$ after admission. According to PICS scores, $>80$ points was classified as non-critically ill group, $70-80$ points was defined as critically ill group, and $<70$ points was the extremely critically ill group. There were 8 cases in the non-critically ill group, 42 cases in the critically ill group, and 30 cases in the extremely critically ill group. Twelve patients died in the critically ill group and 16 patients died in the extremely critically ill group. Patients were separated into 2 groups according to the treatment outcomes: 52 patients were included in the survivors group and 28 patients in the deceased group. Clinical characteristics of children with different prognoses were observed. Changes of $\mathrm{ScvO}_{2}$ and $\mathrm{rScO}_{2}$ were measured at the time of diagnosis (T1), at $24 \mathrm{~h}$ (T2) and $48 \mathrm{~h}$ (T3) after diagnosis. Near-infrared spectroscopy (NIRS) (INVOS3100; Unity Scientific, Milford, MA, USA) was used to monitor the local $\mathrm{rSO}_{2}$, and the $\mathrm{rScO} 2$ was monitored in the forehead of patients. NIRS can be used for non-invasive assessment of $\mathrm{rScO}_{2}$ through the intact skull in human subjects by detecting the mixed transmission intensity of oxyhemoglobin and reduced hemoglobin. NIRS light penetrates easily the human tissue and can enter a few centimeters into the cranial. Special substances in tissues, such as hemoglobin, myoglobin and cytochrome, have wavelength-dependent absorption characteristics, which have a great influence on the transmitted light and the reflected light intensity. The human brain contains $600-1,000 \mathrm{mg}$ of hemoglobin per $100 \mathrm{~g}$ of tissue and is well-suited for monitoring using NIRS. Hemoglobin is the main color base of near-infrared light decay in the brain. When the oxidation state of the body changes, its absorption spectrum changes, resulting in a change in the intensity of the light penetrating the organism. According to this, the oxygenation state of hemoglobin can be detected (8). Dynamic electroencephalogram (EEG) changes were monitored and CT scans were performed after the condition of the patients had improved. Follow-up was continued for 3 months after discharge.
Table I. Baseline characteristics of the patients.

\begin{tabular}{|c|c|}
\hline Index & $\begin{array}{l}\text { Patients } \\
(\mathrm{n}=80)\end{array}$ \\
\hline Age (months) & $16.5 \pm 5.2$ \\
\hline \multicolumn{2}{|l|}{ Sex } \\
\hline Male & 48 \\
\hline Female & 32 \\
\hline \multicolumn{2}{|l|}{ State of consciousness } \\
\hline Lethargy & 23 \\
\hline Light coma & 27 \\
\hline Deep coma & 30 \\
\hline Cases of hyperthermia & 42 \\
\hline \multicolumn{2}{|l|}{ Seizure situation } \\
\hline Systemic seizures & 42 \\
\hline Localized seizures & 38 \\
\hline \multicolumn{2}{|l|}{ EEG situation } \\
\hline Mild to moderate dispersion abnormal number & 44 \\
\hline Severe abnormal number & 36 \\
\hline \multicolumn{2}{|l|}{ Head CT examination } \\
\hline Mild to moderate abnormality & 57 \\
\hline Severe abnormality & 23 \\
\hline \multicolumn{2}{|l|}{ Scores } \\
\hline PICS score & $71.4 \pm 12.6$ \\
\hline GCS score & $7.1 \pm 2.6$ \\
\hline \multicolumn{2}{|l|}{ Treatment outcomes } \\
\hline Survivors & 52 \\
\hline Deceased & 28 \\
\hline
\end{tabular}

EEG, electroencephalogram; PICS, post-intensive care syndrome; GCS, Glasgow Coma Scale.

Statistical analysis. SPSS 21.0 statistical software (IBM Corp., Armonk, NY, USA) was used for the statistical analysis of the data. Measurement data were expressed as mean \pm standard deviation. Count data were expressed as cases (n). Continuous variables were compared using t-test. Categorical variables were compared using Chi-square test or Fisher's test. ANOVA was performed for the comparison between groups. Least Significant Difference was the posthoc test used in our study. $\mathrm{P}<0.05$ was considered to indicate a statistically significant difference.

\section{Results}

Baseline characteristics of the study subjects. The mean age of the patients was $16.5 \pm 5.2$ months, and there were 48 male and 32 female patients. All the children had disturbance of consciousness, including 30 cases of deep coma, 27 cases of shallow coma, and 23 cases of drowsiness. Fever was detected in all patients, and there were 42 cases of hyperthermia and 38 cases of non-hyperthermia. Seizures were observed in all patients, and there were 42 cases of systemic seizures and 38 cases of local seizures. EEG was performed 
Table II. Comparison of clinical features of children with different conditions.

\begin{tabular}{lccccccc}
\hline Groups & & & & & $\begin{array}{c}\text { Severe } \\
\text { abnormalities } \\
\text { of EEG }\end{array}$ & $\begin{array}{c}\text { Severe } \\
\text { abnormalities } \\
\text { of head CT }\end{array}$ & $\begin{array}{c}\text { Survival } \\
\text { cases }\end{array}$ \\
\hline Non-critically ill group & 8 & 4 & 0 & 1 & 1 & 0 & 8 \\
Critically ill group & 42 & 21 & 12 & 22 & 16 & 11 & 30 \\
Extremely critically ill group & 30 & 17 & 18 & 19 & 19 & 12 & 14 \\
$\chi^{2}$ Fisher's value & & 0.409 & 12.709 & 6.411 & 8.085 & 5.216 & 9.503 \\
P-value & 0.896 & 0.001 & 0.038 & 0.015 & 0.078 & 0.009 \\
\hline
\end{tabular}

EEG, electroencephalogram.

Table III. Comparison of clinical features of children with different prognoses.

\begin{tabular}{lcccccccc}
\hline Groups & Cases & Hyperthermia & Deep coma & $\begin{array}{c}\text { Systemic } \\
\text { seizures }\end{array}$ & $\begin{array}{c}\text { Severe } \\
\text { abnormalities } \\
\text { of EEG }\end{array}$ & $\begin{array}{c}\text { Severe } \\
\text { abnormalities } \\
\text { of head CT }\end{array}$ & PICS score & GCS score \\
\hline Survivors group & 52 & 23 & 16 & 22 & 18 & 11 & $77.9 \pm 8.1$ & $8.3 \pm 2.2$ \\
Deceased group & 28 & 19 & 14 & 20 & 18 & 12 & $59.2 \pm 10.4$ & $4.9 \pm 1.6$ \\
$\chi^{2} /$ t value & & 4.074 & 2.872 & 6.189 & 6.474 & 4.185 & 8.930 & 8.145 \\
P-value & 0.061 & 0.146 & 0.019 & 0.018 & 0.068 & $<0.01$ & $<0.01$ \\
\hline
\end{tabular}

EEG, electroencephalogram; PICS, post-intensive care syndrome; GCS, Glasgow Coma Scale.

on the 80 patients, and mild to moderate abnormalities were observed in 44 cases, and severe abnormalities in 36 cases. All patients underwent cranial CT examination. The initial manifestations were diffuse edema of whole brain symmetry, or low density changes and necrosis of the fronto-temporal part, while late manifestations were cranial sulcus enlargement and ventricles enlargement. The number of patients with mild to moderate head CT abnormalities was 57, and the number of patients with severe abnormalities was 23 . Final treatment outcomes for all patients were 52 survivors and 28 deceased children (Table I).

Comparison of clinical features of children with different conditions. PICS scoring was performed on children with sepsis-related encephalopathy immediately after admission. There were 8 cases in the non-critically ill group, 42 cases in the critically ill group, and 30 cases in the extremely critically ill group. Statistical analysis showed that there were no statistically significant differences in hyperthermia and severe brain CT abnormalities among the groups of patients with different disease conditions $(\mathrm{P}>0.05)$. There were statistically significant differences among the 3 groups regarding deep coma, systemic seizures, severe EEG abnormalities and the survival of the children $(\mathrm{P}<0.05)$. The clinical characteristics of children with different conditions are shown in Table II.

Comparison of clinical features of children with different prognoses. Patients were separated into 2 groups according to the treatment outcomes: 52 patients in the survivors group and 28 patients in the deceased group. Statistical analysis showed that hyperthermia, severe abnormalities of head CT and deep coma were not associated with patients' survival $(\mathrm{P}>0.05)$. Systemic seizures, severe EEG abnormalities, PICS scores and GCS scores were significantly associated with the prognosis of patients $(\mathrm{P}<0.05)($ Table III).

Comparison of $\mathrm{rScO}_{2}$ and $\mathrm{ScvO}_{2}$ in children with different prognoses. The clinical characteristics of children with different prognoses were observed. Changes of $\mathrm{ScvO}_{2}$ and $\mathrm{rScO}_{2}$ at $\mathrm{T} 1, \mathrm{~T} 2$ and $\mathrm{T} 3$ were measured. Results showed that $\mathrm{rScO}_{2}$ values in the deceased group were significantly lower than those in the survivors group at the different time-points, but the differences were not significant $(\mathrm{P}>0.05)$. On the contrary, $\mathrm{ScvO}_{2}$ values were significantly higher in the deceased group than in the survivors group, and the differences were statistically significant $(\mathrm{P}<0.05)$. There was no statistically significant difference in the changes of $\mathrm{rScO}_{2}$ and $\mathrm{ScvO}_{2}$ at the different time-points in the same group ( $\mathrm{P}>0.05)$. Post-hoc test was used to compare the changes of $\mathrm{rScO}_{2}$ and $\mathrm{ScvO}_{2}$ at two different time-points, and the results showed that the differences were not statistically significant $(\mathrm{P}>0.05)$ (Table IV).

Treatment and follow-up results. After active rescue, some patients' consciousness gradually recovered, including recovery in $<24 \mathrm{~h}$ in 5 cases, $24-72 \mathrm{~h}$ in 12 cases, $>72 \mathrm{~h}$ to 1 week 
Table IV. Comparison of $\mathrm{rScO}_{2}$ and $\mathrm{ScvO}_{2}$ in children with different prognoses.

\begin{tabular}{|c|c|c|c|c|c|c|c|}
\hline \multirow[b]{2}{*}{ Groups } & \multirow[b]{2}{*}{ Cases } & \multicolumn{2}{|c|}{$\mathrm{T} 1$} & \multicolumn{2}{|c|}{$\mathrm{T} 2$} & \multicolumn{2}{|c|}{$\mathrm{T} 3$} \\
\hline & & $\mathrm{rScO}_{2}$ & $\mathrm{ScvO}_{2}$ & $\mathrm{rScO}_{2}$ & $\mathrm{ScvO}_{2}$ & $\mathrm{rScO}_{2}$ & $\mathrm{ScvO}_{2}$ \\
\hline Survivors group & 52 & $63.6 \pm 10.9$ & $70.9 \pm 9.8$ & $62.3 \pm 11.3$ & $72.1 \pm 9.1$ & $60.3 \pm 11.0$ & $69.2 \pm 11.9$ \\
\hline Deceased group & 28 & $59.3 \pm 10.5$ & $76.3 \pm 10.2$ & $59.6 \pm 10.9$ & $76.5 \pm 10.0$ & $58.7 \pm 9.2$ & $75.2 \pm 8.5$ \\
\hline t value & & 1.679 & -2.320 & 1.019 & -1.998 & 0.637 & -2.584 \\
\hline P-value & & 0.097 & 0.023 & 0.311 & 0.049 & 0.526 & 0.012 \\
\hline
\end{tabular}

$\mathrm{rScO}_{2}$, regional cerebral oxygen saturation; $\mathrm{ScvO}_{2}$, central venous oxygen saturation.

in 34 cases, and $>1$ week in 1 case. Seizure symptoms in children treated with antiepilepticism were gradually controlled within 1 week. At the time of discharge, there were 37 cases of normal neurological function and 15 cases of abnormal neurological function. Among them, there were 2 cases of mild hearing impairment, 3 cases of mild exercise retardation, and 2 cases of mild decrease of muscle tension. The 3 months follow-up in the 52 cases of the surviving children showed that the neurological functions were completely recovered. Cranial image (CT or MRI) was normal in 45 cases, and abnormal in 7 cases. The 7 abnormal cases manifested as the widening of sulcus and a sign of brain atrophy with enlarged ventricles. EEG was performed on all 52 cases during follow-up, and 38 cases were normal, while 14 cases were mildly abnormal.

\section{Discussion}

Sepsis-related encephalopathy refers to the central nervous system dysfunction caused by sepsis, with no effective treatment, and Papadopoulos et al have pointed out that the incidence of septic brain disease was $8-70 \%$ (9). Wilson and Young (10) redefined the brain dysfunction caused by sepsis as septic-related encephalopathy, which was a very common disease in PICU with a high mortality rate and high hospitalization cost, that was often seen in children. Pathogenesis of septic encephalopathy remains unclear. At present, it is believed that the pathogenesis of septic encephalopathy mainly includes inflammatory mediator release, impaired blood-brain barrier, mitochondrial dysfunction, neurotransmitter changes and cell apoptosis. Terborg et al (11) have pointed out that vasomotor dysfunction which was regarded as the main cause of sepsis-associated encephalopathy, often leads to dysregulation of cerebral blood flow and can directly affect brain function and cerebral blood circulation. Studies have shown that patients with sepsis have relatively high levels of aromatic amino acids in plasma, which can easily lead to the production of pseudo-neurotransmitters, affect neuronal function, and cause symptoms and signs of various neurological impairments including disturbance of consciousness $(12,13)$. Sharshar et al (14) performed pathological examination on the brain tissue of patients who died of sepsis and found that the incidence of microabscess was high and microabscess may be one of the causes of sepsis-associated encephalopathy. In addition, many substances, such as IL,
TNF, Glu, and NO can act on the nervous system in different ways, causing corresponding damage to the nervous system. Moreover, improper use of drugs and infusion can also induce iatrogenic brain injury.

The high incidence and mortality of sepsis-associated encephalopathy have attracted closer attention from scholars. In sepsis-associated encephalopathy is the sepsis complication which increases mortality. Eidelman et al (15) comprehensively elaborated and analyzed the definition, etiology and mortality of sepsis-associated encephalopathy and found that the mortality of septic encephalopathy was 33-39\%. At present, APACHE II scoring system is generally used in ICU to assess the severity of illness of adult patients. PICS scoring system is used for the assessment of pediatric patients because of their special characteristics. PICS scores include multiple indicators, such as vital signs, $\mathrm{pH}$, electrolytes, renal function, and gastrointestinal function. However, PICS scoring does not include neurological assessment. GCS score can supplement and improve the PICS score to some extent. Therefore, this study combined the two scoring systems to assess the disease condition. In this study 80 children admitted to the Children's Hospital Affiliated to Zhengzhou University with sepsis-associated encephalopathy were assigned. The final treatment outcomes were 52 survivors and 28 deceased, with a survival rate of $65 \%$. PICS scoring was performed on children with sepsis-related encephalopathy immediately after admission and patients were separated into different groups according to their scores. It was observed that deep coma, general seizures, severe EEG abnormalities, and the survival of children were significantly associated with the severity of the disease. Therefore, we can assess patient's condition by observing patient's state of consciousness, epileptic seizures and EEG changes. Children were separated into the survivors and the deceased groups according to the treatment outcomes. The systemic seizures, severe EEG abnormalities, PICS and GCS scores were significantly associated with the prognosis of patients. Therefore, patient's prognosis can be assessed through seizures and EEG changes in the patients. PICS and GCS scores are important for evaluating the prognosis of children. The lower the PICS score, the more severe the clinical manifestations of the disease in children, and the higher the mortality rate. Therefore, PCIS and GCS scores are of great significance in assessing the severity of critically ill children.

The current treatment for sepsis patients is mainly early recovery, control of the source of infection and empirical 
anti-infective treatment. Pathological changes in sepsis are often manifested as cellular hypoxia caused by vascular reactivity. Therefore, maintaining hemodynamic stability is crucial for children with sepsis. Under stable respiratory conditions, $\mathrm{ScvO}_{2}$ can reflect the body's oxygen supply and demand balance. By assessing tissue oxygenation in children with septic-associated encephalopathy, NIRS can monitor $\mathrm{rScO}_{2}$ changes, and to some extent reflect the changes in brain metabolism. A study of 60 patients with septic shock has shown that early monitoring of patient's $\mathrm{ScvO}_{2}$ to guide the fluid resuscitation of septic shock can effectively reduce the incidence and mortality of multiple organ dysfunction syndrome, which is conducive of improving the prognosis of patients (16). In the present study, changes of $\mathrm{ScvO}_{2}$ and $\mathrm{rScO}_{2}$ were measured at the T1, T2 and T3 time-points. The results revealed that the $\mathrm{ScvO}_{2}$ values in the deceased group were significantly higher than those in the survivors group, at all different time-points, and the differences were statistically significant, suggesting that changes in $\mathrm{ScvO}_{2}$ are closely related to the prognosis of children. The treatment of children with septic-related encephalopathy can be assisted by continuous monitoring of $\mathrm{ScvO}_{2}$. It is beneficial to the prognosis of children and is worthy of clinical application.

At present, EEG is widely used in the diagnosis of patients with sepsis-associated encephalopathy, and the sensitivity is high (17). Although some patients with septic brain disease have no nervous system dysfunction, the mild diffuse abnormal waveform can usually be observed through EEG. In this study, electroencephalography was performed on all 80 cases and 44 patients showed mild to moderate abnormalities, 36 patients showed severe abnormalities, and of the 28 deceased cases, 18 had severe abnormalities of EGG, indicating that the prognosis of patients with septic-related encephalopathy is significantly related to changes in EEG. During follow-up, the conditions improved in some patients. However, there were still some children with abnormal EEG. Therefore, EGG is of great significance for the evaluation of sepsis-associated encephalopathy. EEG can help not only the early diagnosis of children but also the assessment of the condition and prognosis during treatment. CT can show any changes in the head of children with sepsis, such as narrowing of the ventricle, sulci, and cisterna, unclear gray matter, local low-density shadow or even severe liquefaction necrosis. In the recovery period, the head CT showed mild to moderate brain atrophy. In this study, in the early stages of the disease, the head CT showed diffuse edema of the whole brain, that is, narrowing of the sulci, ventricles, cisterns, or unclear boundaries of gray matter. It was found that during the recovery and 3 months follow-up most patients returned to normal, but some children still showed signs of brain atrophy with widening of the sulcus and ventricles. The results of this study indicate that head CT can be used to assess brain damage, assist clinical treatment and evaluate the prognosis. Patients with sepsis-associated encephalopathy generally have severe symptoms, high mortality and poor prognosis. For surviving patients, nervous system damage can be gradually repaired. In this study, the consciousness in the majority of the children was gradually recovered within 1 week. The convulsive symptoms of the children treated with antiepileptic therapy were gradually controlled within 1 week. After 3 months of follow-up, the neurological examination showed that the nervous system of all the surviving children returned to normal, suggesting that there is still a lot of room for the neurological rehabilitation of sepsis related encephalopathy if the children can survive the acute stage safely. However, this study has some limitations. Firstly, this study is a retrospective study. Exclusion diagnosis was adopted for the diagnosis of sepsis-related encephalopathy. If we could prospectively use EEG, somatosensory evoked potentials combined with imaging to diagnose sepsis-related encephalopathy, the incidence statistics could be more accurate. Secondly, this study is a single-center survey. The sample size collected in a short time is relatively small, and the conclusions obtained cannot fully reflect the overall level of China. As a result, a large-scale multi-center epidemiological survey is needed.

Collectively, this study shows that the lower the PICS and GCS scores of children with sepsis-related encephalopathy are, the more severe the disease is, and the more likely they are to suffer from consciousness disorder, epileptic seizure and abnormal EEG changes. The change of $\mathrm{ScvO}_{2}$ is closely related to the prognosis of children. Therefore, for children with sepsis-related encephalopathy, continuous monitoring of their consciousness, seizures, EEG and $\mathrm{ScvO}_{2}$ changes can be used to evaluate the treatment status and prognosis of children, which is worthy of clinical promotion.

\section{Acknowledgements}

Not applicable.

\section{Funding}

The present study was supported by 2018 Henan Science and Technology Development Plan Project (Project name: 'Study on the changes of cerebral hemoglobin concentration index and cerebral oxygen saturation in children with septic shock', no. 182102310427).

\section{Availability of data and materials}

The datasets used and/or analyzed during the present study are available from the corresponding author on reasonable request.

\section{Authors' contributions}

JG conceived and designed this study. YC, QW and JS acquired and analyzed the data. LC and ZJ were involved in the design of the study, drafted, revised and finalized the manuscript. All authors read and approved the final manuscript.

\section{Ethics approval and consent to participate}

The study was approved by the Ethics Committee of the Children's Hospital Affiliated to Zhengzhou University (Zhengzhou, China). Patients who participated in this research had complete clinical data and signed informed consents were obtained from their parents.

\section{Patient consent for publication}

Not applicable. 


\section{Competing interests}

The authors declare that they have no competing interests.

\section{References}

1. Shen HN, Lu CL and Yang HH: Epidemiologic trend of severe sepsis in Taiwan from 1997 through 2006. Chest 138: 298-304, 2010.

2. Anas AA, Wiersinga WJ, de Vos AF and van der Poll T: Recent insights into the pathogenesis of bacterial sepsis. Neth J Med 68: 147-152, 2010.

3. Semeraro N, Ammollo CT, Semeraro F and Colucci M: Sepsis, thrombosis and organ dysfunction. Thromb Res 129: 290-295, 2012.

4. Angus DC and van der Poll T: Severe sepsis and septic shock. N Engl J Med 369: 2063-2063, 2013.

5. Thabet FC, Zahraa JN and Chehab MS: Adherence to surviving sepsis guidelines among pediatric intensivists. A national survey. Saudi Med J 38: 609-615, 2017.

6. Artigas A, Carlet J, Martin-Loeches I, Niederman M and Torres A: 23rd International Symposium on Infections in the Critically Ill Patient. Med Sci (Basel) 6: 1-57, 2018.

7. Souza DC, Brandão MB and Piva JP: From the International Pediatric Sepsis Conference 2005 to the Sepsis-3 Consensus. Rev Bras Ter Intensiva 30: 1-5, 2018 (In Portuguese).

8. Hirtz DG: Report of the National Institute of Neurological Disorders and Stroke workshop on near infrared spectroscopy. Pediatrics 91: 414-417, 1993.

9. Papadopoulos MC, Davies DC, Moss RF, Tighe D and Bennett ED: Pathophysiology of septic encephalopathy: A review. Crit Care Med 28: 3019-3024, 2000.
10. Wilson JX and Young GB: Progress in clinical neurosciences: sepsis-associated encephalopathy: Evolving concepts. Can J Neurol Sci 30: 98-105, 2003.

11. Terborg C, Schummer W, Albrecht M, Reinhart K, Weiller C and Röther J: Dysfunction of vasomotor reactivity in severe sepsis and septic shock. Intensive Care Med 27: 1231-1234, 2001.

12. Basler T, Meier-Hellmann A, Bredle D and Reinhart K: Amino acid imbalance early in septic encephalopathy. Intensive Care Med 28: 293-298, 2002

13. Nakamura T, Kawagoe Y, Matsuda T, Ebihara I and Koide H: Effects of polymyxin B-immobilized fiber hemoperfusion on amino acid imbalance in septic encephalopathy. Blood Purif 21: 282-286, 2003

14. Sharshar T, Gray F, Lorin de la Grandmaison G, Hopkinson NS, Ross E, Dorandeu A, Orlikowski D, Raphael JC, Gajdos P and Annane D: Apoptosis of neurons in cardiovascular autonomic centres triggered by inducible nitric oxide synthase after death from septic shock. Lancet 362: 1799-1805, 2003.

15. Eidelman LA, Putterman D, Putterman C and Sprung CL: The spectrum of septic encephalopathy. Definitions, etiologies, and mortalities. JAMA 275: 470-473, 1996.

16. Agarwal S, Cox AJ, Herrington DM, Jorgensen NW, Xu J, Freedman BI, Carr JJ and Bowden DW: Coronary calcium score predicts cardiovascular mortality in diabetes: Diabetes heart study. Diabetes Care 36: 972-977, 2013.

17. Tong DM, Zhou YT, Wang GS, Chen XD and Yang TH: Early prediction and outcome of septic encephalopathy in acute stroke patients with nosocomial coma. J Clin Med Res 7: 534-539, 2015.

This work is licensed under a Creative Commons

Attribution-NonCommercial-NoDerivatives 4.0 International (CC BY-NC-ND 4.0) License. 